\title{
Discovery of anticancer clinical candidate, tosedostat, as an analgesic agent
}

\author{
Rohit Singh*, Wei Xie, Jessica Williams, Robert Vince*, Swati S. More*
}

\author{
Center for Drug Design, \\ Academic Health Center, \\ College of Pharmacy, \\ University of Minnesota, \\ Minneapolis, MN-55455, USA.
}

Corresponding Authors:

Rohit Singh: $\quad$ singh109@umn.edu; +1 (612) 624-2146

Robert Vince: $\quad$ vince001@umn.edu; +1 (612) 624-9911

Swati S. More: $\quad$ morex002@umn.edu; +1 (612) 626-1660

\section{Supporting Information}

\section{Table of Contents}

Figure S1. Dose-Response Curves for the Determination of $\mathbf{E D}_{\mathbf{5 0}}$ values of tosedostat........................S2

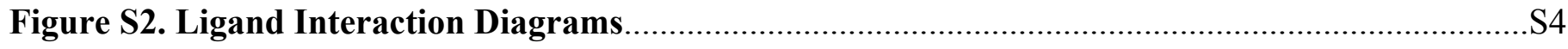

Figure S3. Batimastat Docked in the Binding Site of Aminopeptidase N...........................................S6

Figure S4. Batimastat Docked in the Binding Site of Neprilysin.......................................................S7

Figure S5. Docking Poses with Ligand Surface Area for Tosedostat Docked in APN and NEP..........S8

Figure S6. Docking Poses with Ligand Surface Area for Batimastat Docked in APN and NEP...........S9

Figure S7. Prolonged Time-dependent Antinociceptive Effects of Tosedostat in Hot-plate Assay....S10

Table S1. Determination of the Formation of CHR-79888 in APN and NEP Assays..........................S11 
Figure S1: Dose-response curves for the determination of $\mathrm{ED}_{50}$ values of tosedostat at various time-points: (A) 15 minutes, (B) 30 minutes, (C) 45 minutes, (D) 60 minutes
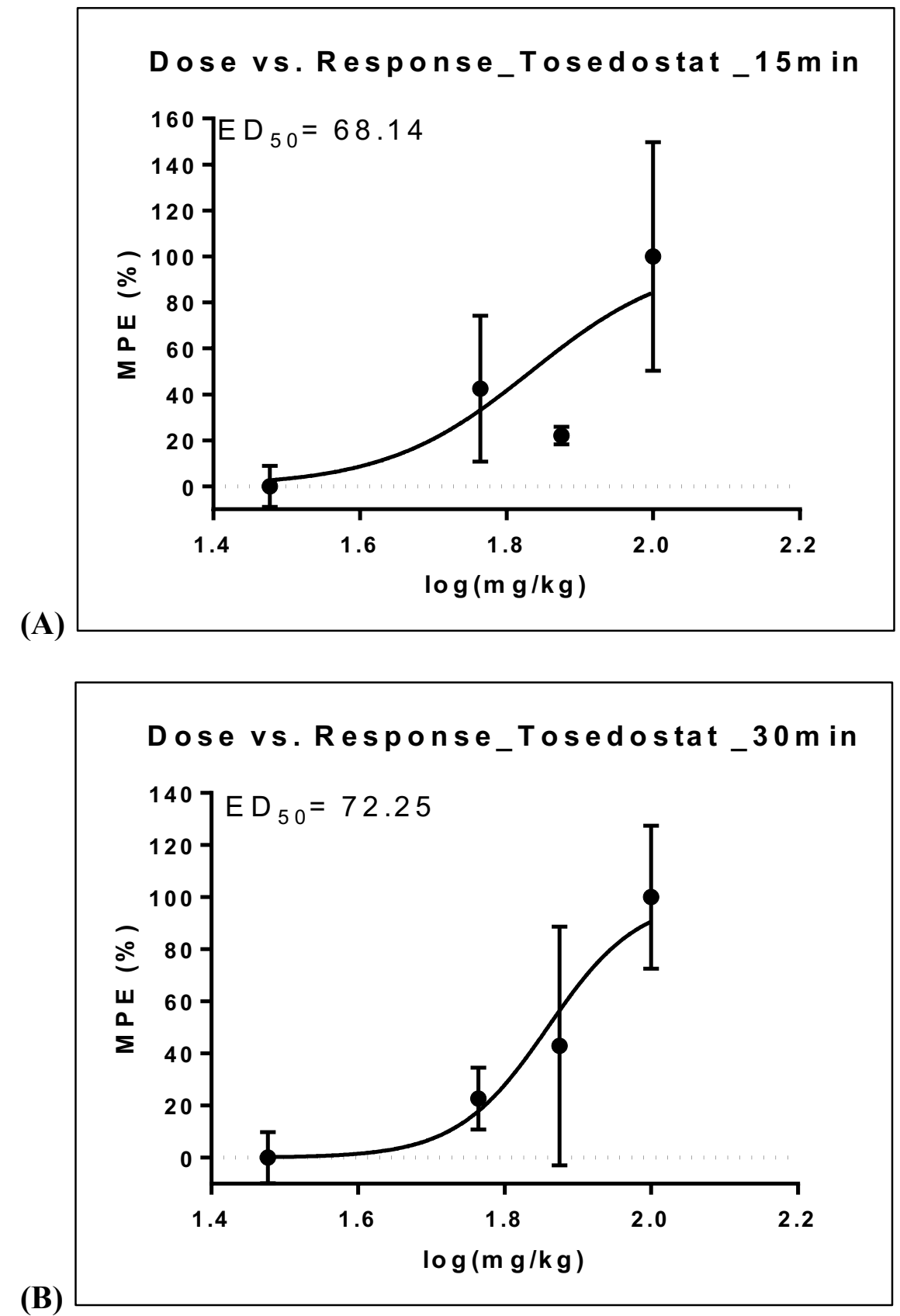


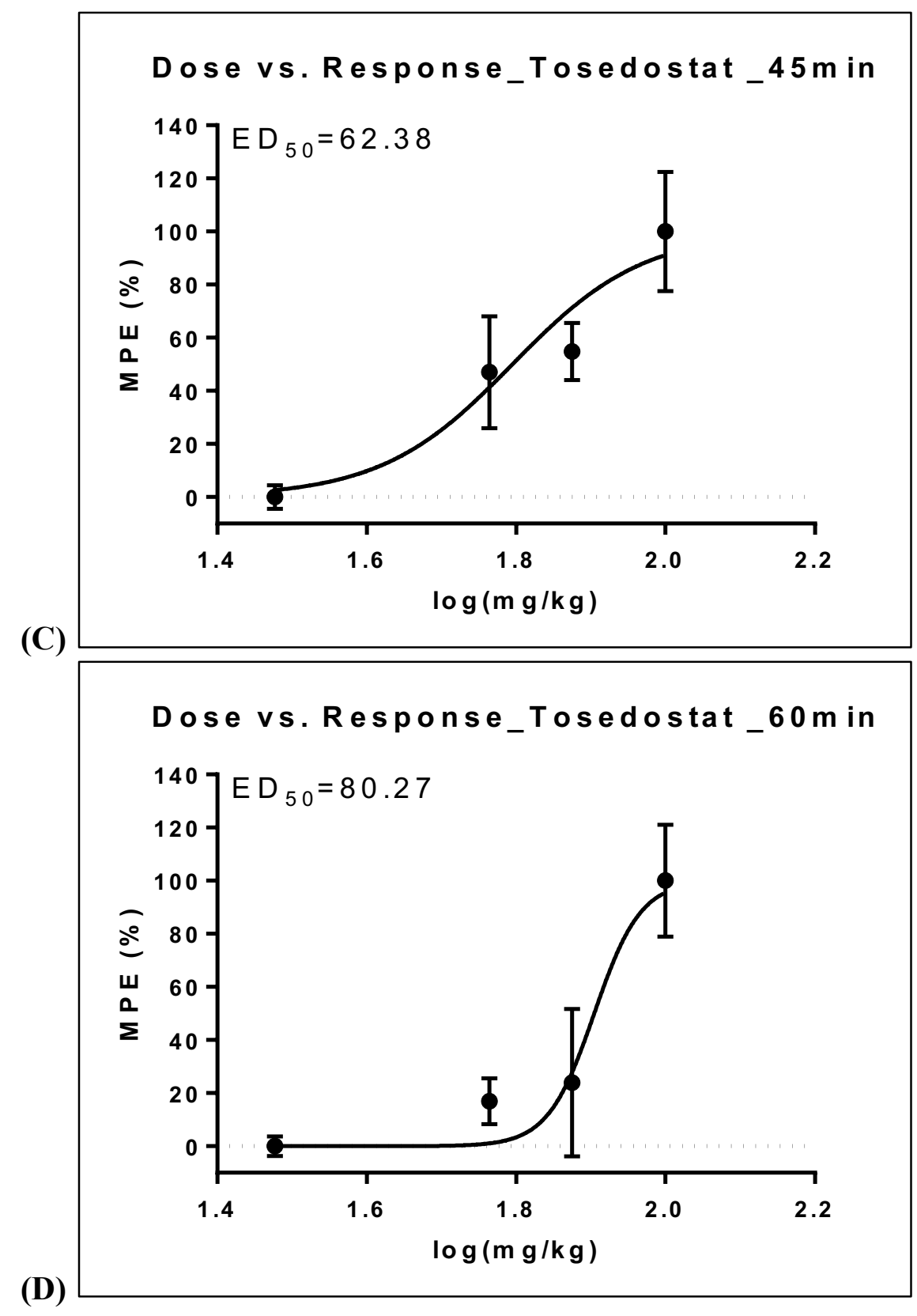


Figure S2. Ligand Interaction Diagrams of: (A) Tosedostat docked in the active site of APN (B) Tosedostat docked in the active site of NEP (C) Batimastat docked in the active site of APN (D) Batimastat docked in the active site of NEP. Various colors in the LID represent diverse charge properties of the amino acids: green - hydrophobic residues, cyan - polar residues, blue - charged residues (positive), red - charged residues (negative). Zinc metal is portrayed in gray.

(A)

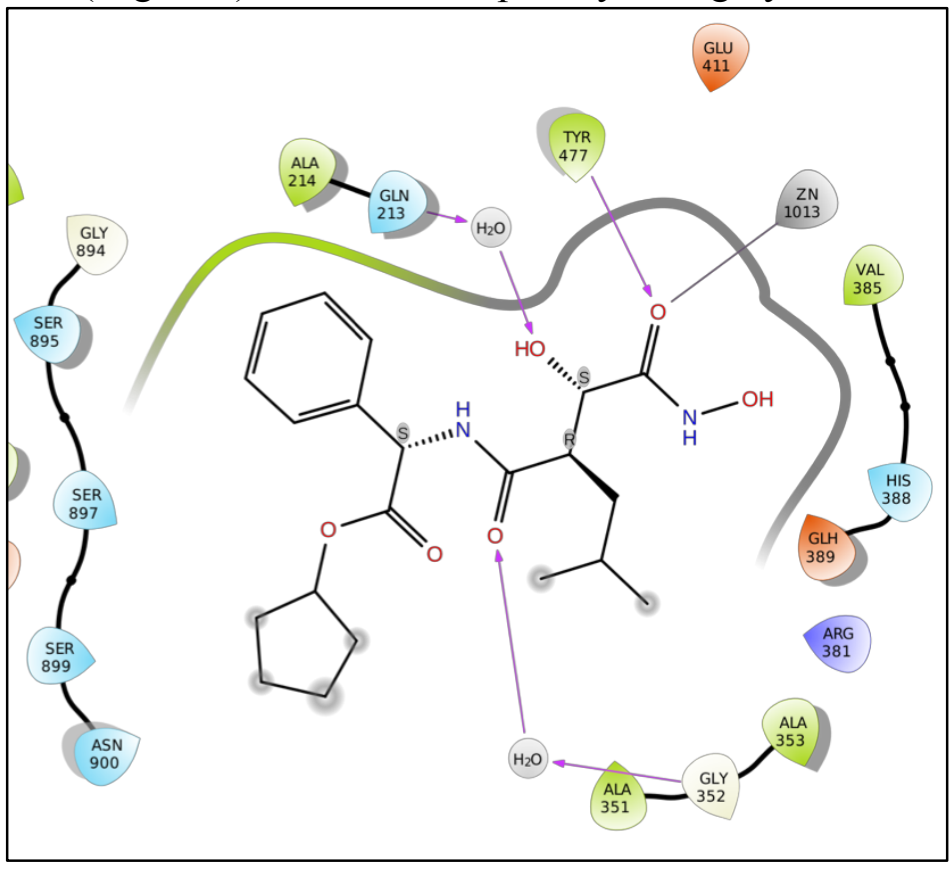

(B)

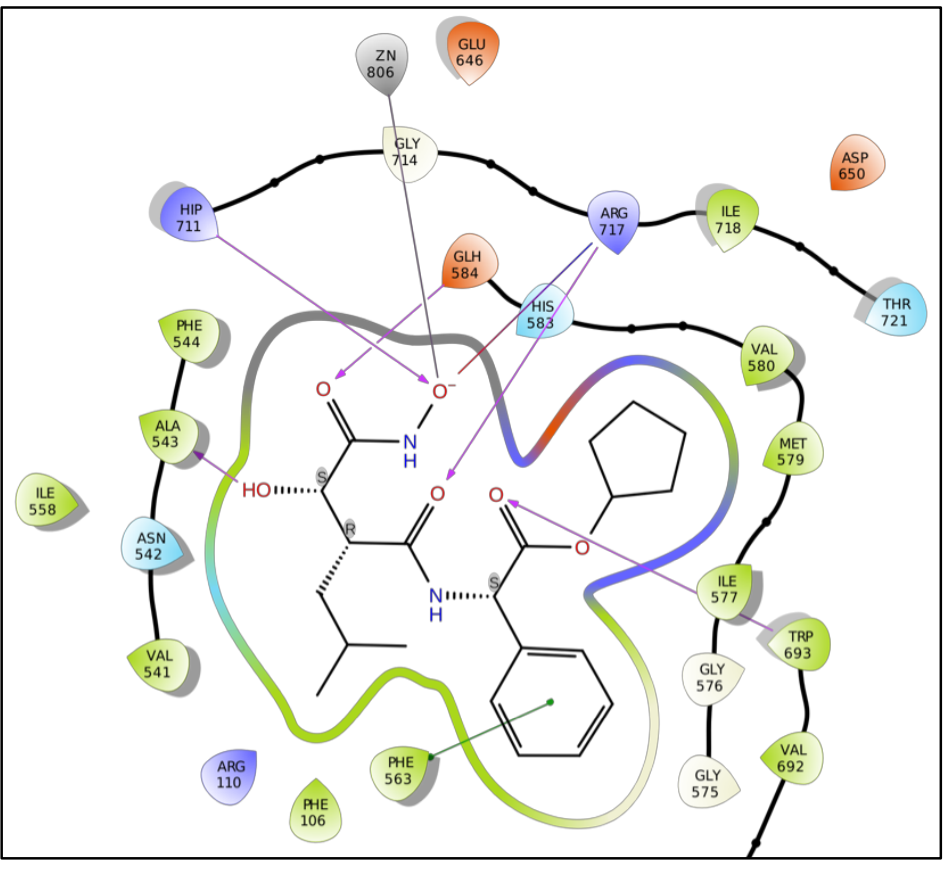



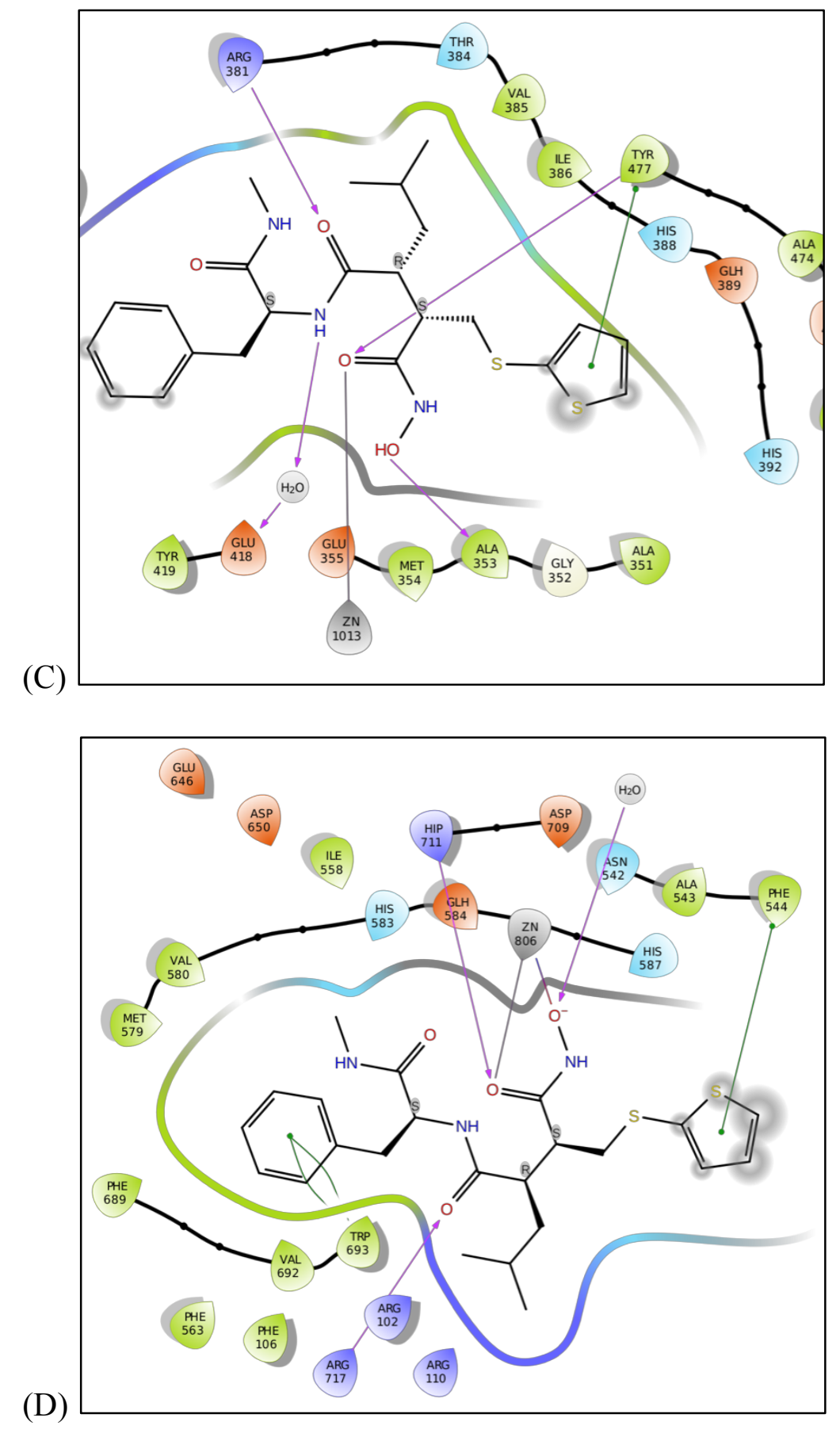
Figure S3. Batimastat (teal ball and stick) docked in the binding site of aminopeptidase N - PDB code 4FYR. $\mathrm{Zn}^{2+}$ cation is depicted as a green sphere. The protein is depicted as yellow (stick). Blue, red, and orange atoms represent nitrogen, oxygen, and sulphur respectively. All residues are labeled in accordance with the reported crystal structures from the respective PDB files of the enzymes. Dashed lines represent Ligand-Receptor interactions: Red dashed line for $\mathrm{H}$-bond interactions, orange for aromatic- $\mathrm{H}$ bond interactions, cyan for salt-bridge, and pink for $\pi-\pi$ stacking interactions. Hydrogen atoms from the ligand and the receptor grid have been removed for clarity.

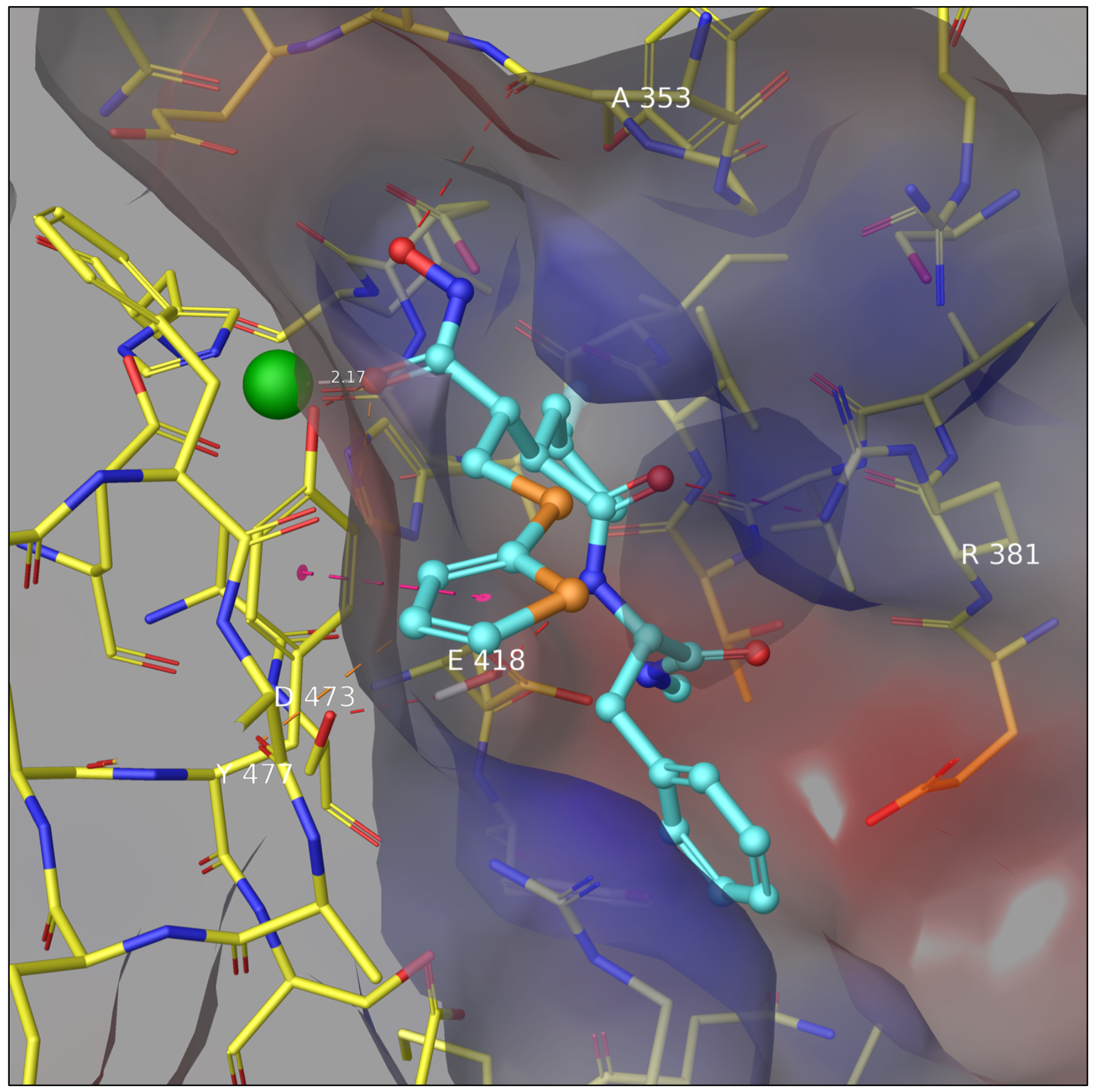


Figure S4. Batimastat (teal ball and stick) docked in the binding site of neprilysin - PDB code 5JMY. $\mathrm{Zn}^{2+}$ cation is depicted as a green sphere. The protein is depicted as yellow (stick). Blue, red, and orange atoms represent nitrogen, oxygen, and sulphur respectively. All residues are labeled in accordance with the reported crystal structures from the respective PDB files of the enzymes. Dashed lines represent LigandReceptor interactions: Red dashed line for $\mathrm{H}-$ bond interactions, orange for aromatic- $\mathrm{H}$ bond interactions, cyan for salt-bridge, and pink for $\pi-\pi$ stacking interactions. Hydrogen atoms from the ligand and the receptor grid have been removed for clarity.

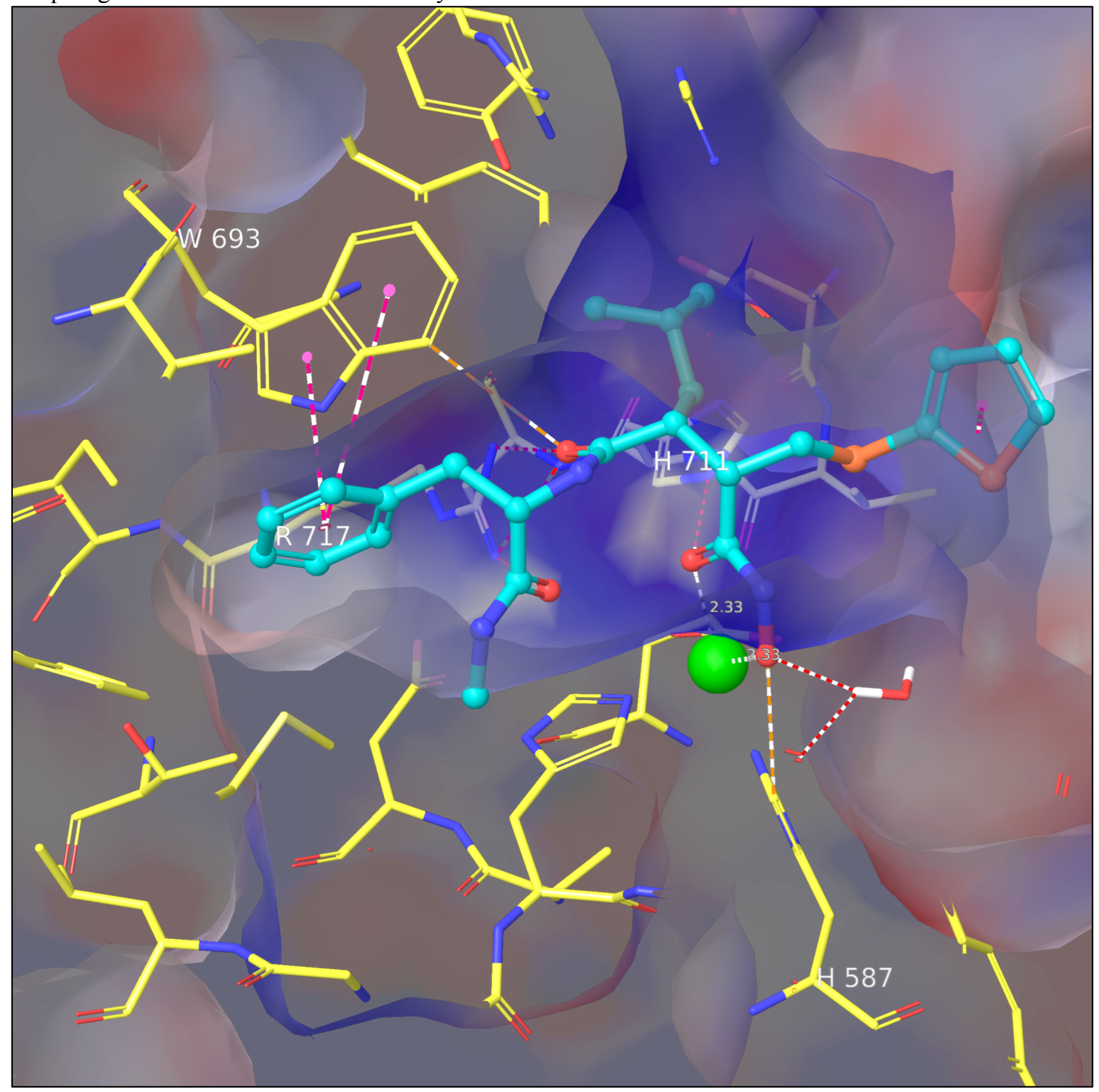


Figure S5. Ligand Surface Area (light orange mesh): $\mathrm{Zn}^{2+}$ cation is depicted as a green sphere. The protein is depicted as yellow (stick). Blue and red atoms represent nitrogen and oxygen respectively. All residues are labeled in accordance with the reported crystal structures from the respective PDB files of the enzymes. Dashed lines represent Ligand-Receptor interactions: Red dashed line for H-bond interactions, orange for aromatic- $\mathrm{H}$ bond interactions, cyan for salt-bridge, and pink for $\pi-\pi$ stacking interactions. Hydrogen atoms from the ligand and the receptor grid have been removed for clarity.

(A) Ligand Surface Area (light orange mesh): Tosedostat (magenta ball and stick) docked in the binding site of aminopeptidase $\mathrm{N}$ - PDB code 4FYR.

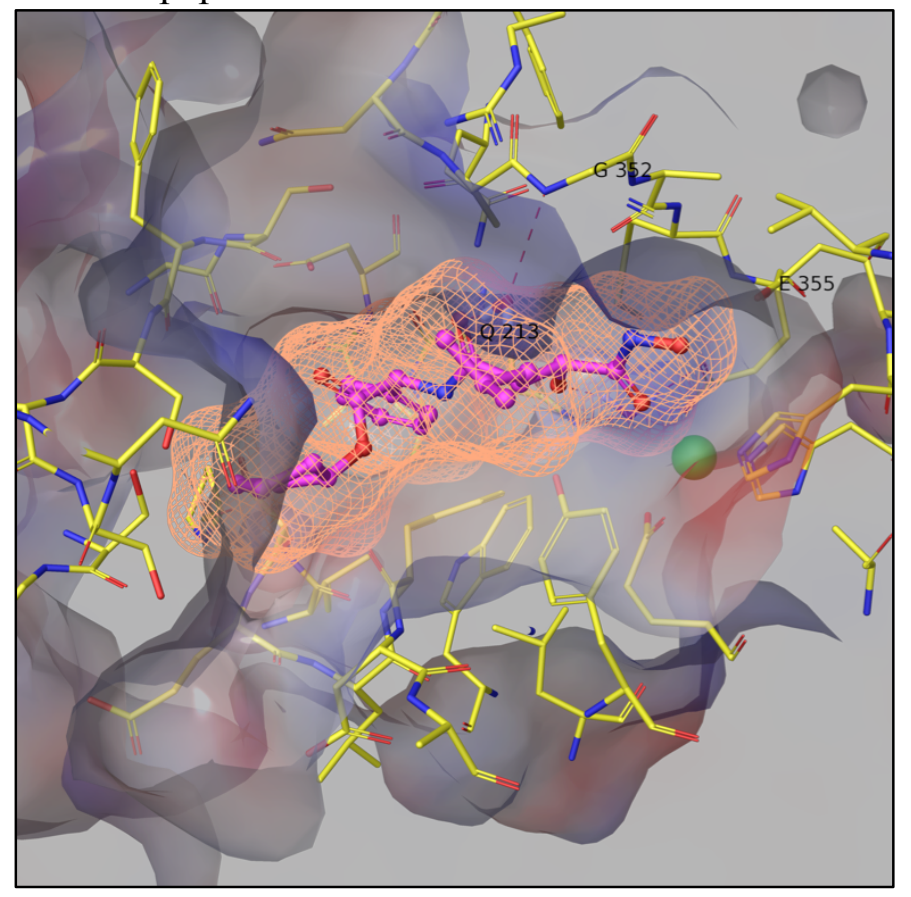

(B) Ligand Surface Area (light orange mesh): Tosedostat (magenta ball and stick) docked in the binding site of neprilysin - PDB code 5JMY.

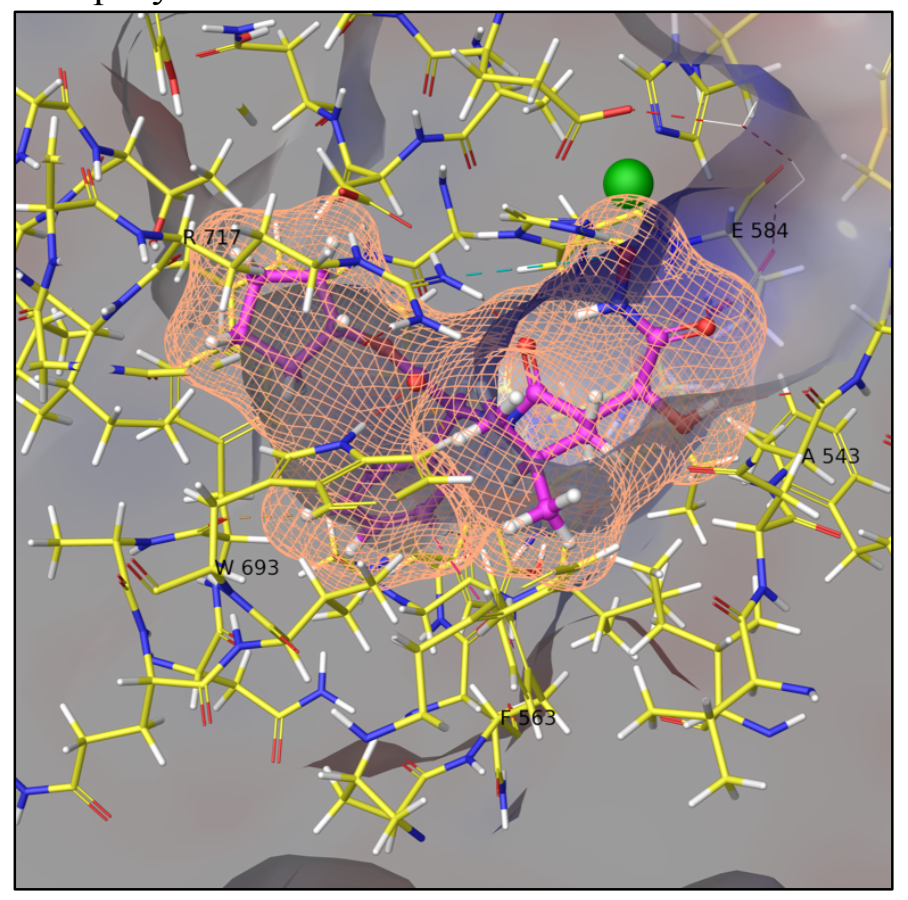


Figure S6. Ligand Surface Area (light teal mesh): $\mathrm{Zn}^{2+}$ cation is depicted as a green sphere. The protein is depicted as yellow (stick). Blue, red, and orange atoms represent nitrogen, oxygen, and sulphur respectively. All residues are labeled in accordance with the reported crystal structures from the respective PDB files of the enzymes. Dashed lines represent Ligand-Receptor interactions: Red dashed line for H-bond interactions, orange for aromatic-H bond interactions, cyan for salt-bridge, and pink for $\pi-\pi$ stacking interactions. Hydrogen atoms from the ligand and the receptor grid have been removed for clarity.

(A) Ligand Surface Area (light teal mesh): Batimastat (teal ball and stick) docked in the binding site of aminopeptidase $\mathrm{N}-\mathrm{PDB}$ code $4 \mathrm{FYR}$.

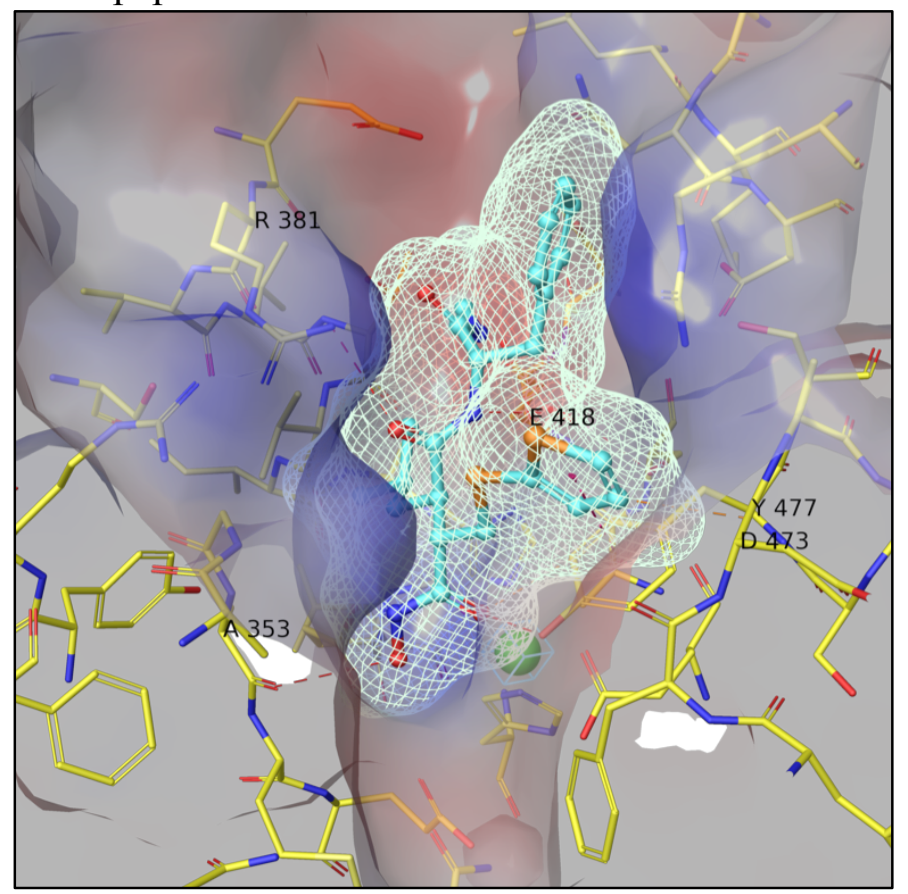

(B) Ligand Surface Area (light teal mesh): Batimastat (teal ball and stick) docked in the binding site of neprilysin - PDB code 5JMY.

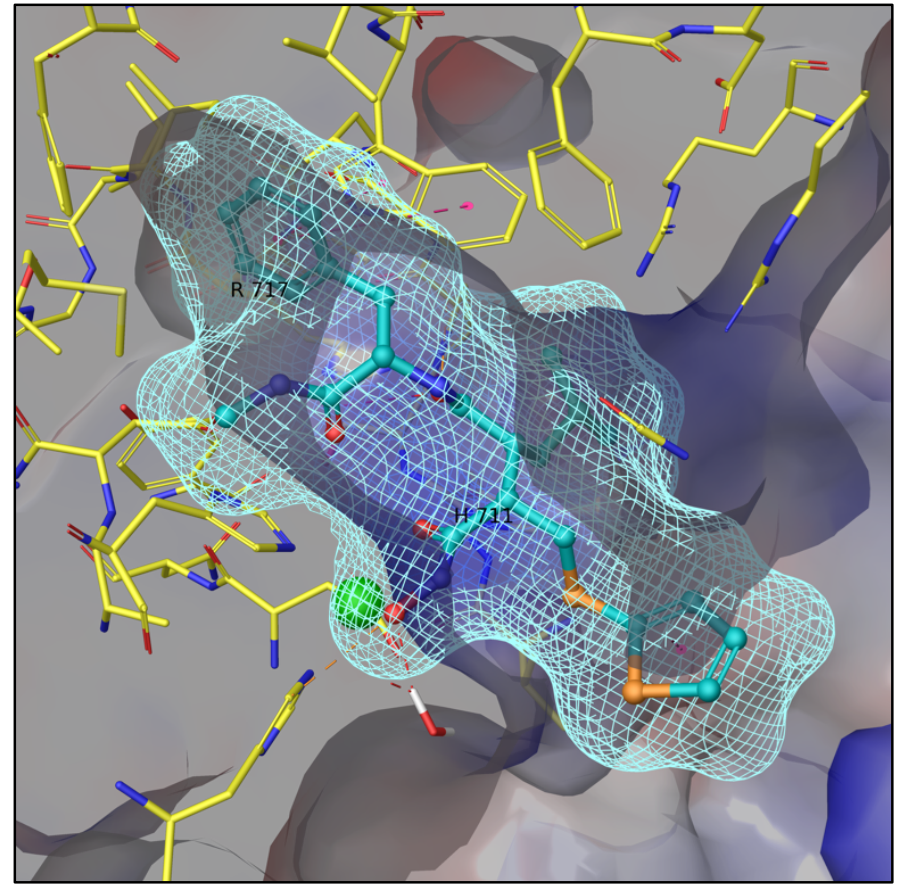


Figure S7. Evaluation of prolonged time-dependent antinociceptive effects of tosedostat in mouse hot-plate assay. The test compound was dissolved in 50\% saline in DMSO solution. Comparison of intraperitoneally delivered tosedostat $(\square)$ and vehicle control $(\square)$. The y-axis represents the effect of the dose expressed in percentage of maximum possible effect (\%MPE). Error bars represent \pm SEM for each time-point. For tosedostat and the vehicle, $\mathrm{n}=3$ animals $/$ time-point. Dosage for i.p. delivery $=58.1 \mathrm{mg} / \mathrm{kg}$ each mouse.

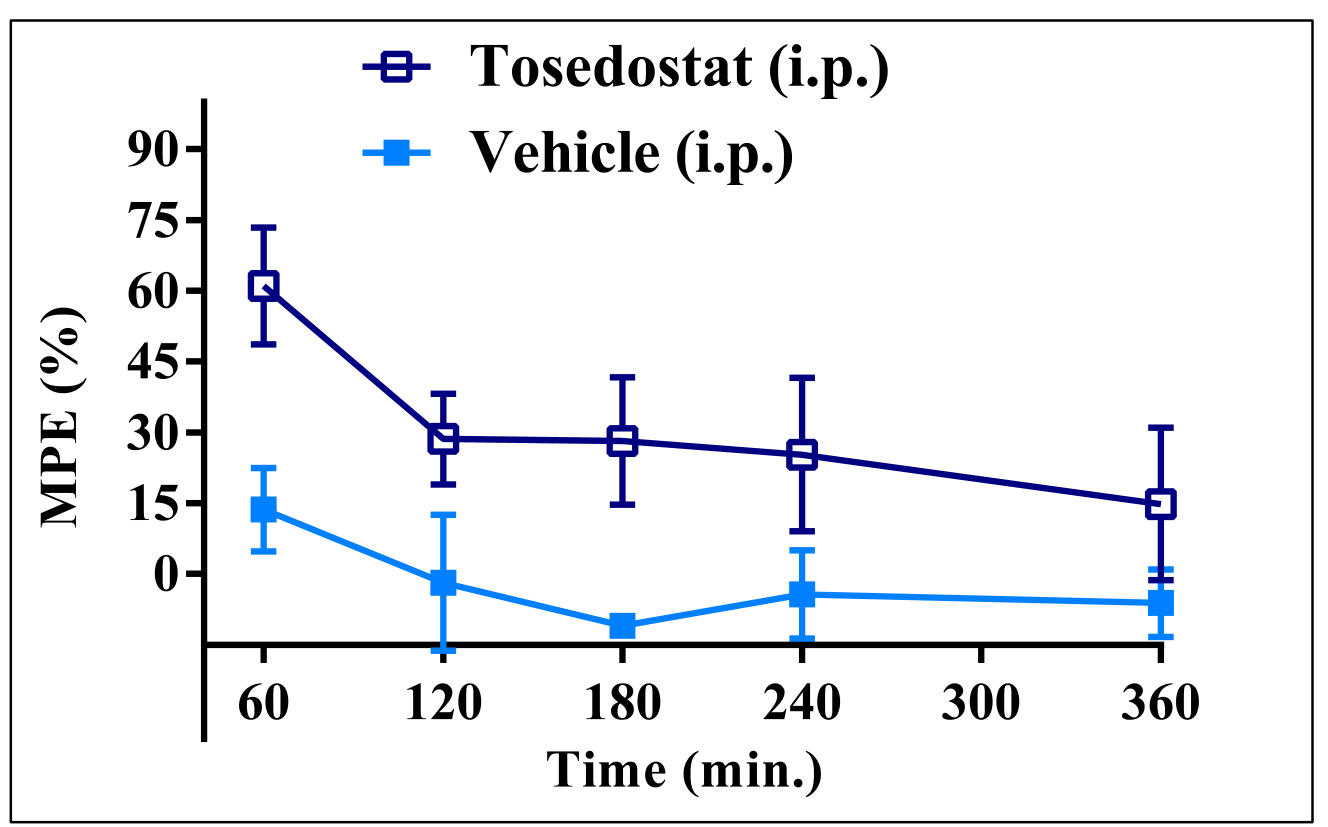


Table S1. Experimental detection of tosedostat (CHR-2797) and its acid form (CHR-79888) in the replicated assay conditions for APN and NEP enzymatic assays. The $\mathrm{T}_{0}$ and $\mathrm{T}_{30}$ values refer to the samples quenched at time $=0$ minutes and time $=30$ minutes, respectively. The samples were analyzed via $\mathrm{LC}-$ MS/MS.

\begin{tabular}{|c|l|c|c|}
\hline $\begin{array}{c}\text { Sample } \\
\text { No. }\end{array}$ & \multicolumn{1}{|c|}{ Sample } & $\begin{array}{c}\text { Tosedostat } \\
(\mathbf{C H R}-\mathbf{2 7 9 7})\end{array}$ & $\begin{array}{c}\text { Tosedostat Acid Form } \\
\text { (CHR-79888) }\end{array}$ \\
\hline 1. & APN_Buffer & $1.52 \mathrm{E}+06$ & Not Detected \\
\hline 2. & APN_ $\mathrm{T}_{0}$ & $1.53 \mathrm{E}+06$ & Not Detected \\
\hline 3. & APN_ $\mathrm{T}_{30}$ & $1.53 \mathrm{E}+06$ & Not Detected \\
\hline 4. & NEP_Buffer & $1.32 \mathrm{E}+06$ & Not Detected \\
\hline 5. & NEP_ $\mathrm{T}_{0}$ & $1.66 \mathrm{E}+06$ & Not Detected \\
\hline 6. & NEP_ $\mathrm{T}_{30}$ & $2.92 \mathrm{E}+06$ & Not Detected \\
\hline
\end{tabular}

